\title{
Preoperative hyperlactatemia and early mortality after liver transplantation: selection of important variables using random forest survival analysis
}

\section{Yuseon Cheong ${ }^{1,2}$, Sangho Lee ${ }^{1}$, Do-Kyeong Lee', Kyoung-Sun Kim ${ }^{1}$, Bo-Hyun Sang ${ }^{3}$, and Gyu-Sam Hwang ${ }^{1}$}

1 Department of Anesthesiology and Pain Medicine, Laboratory for Cardiovascular Dynamics, Asan Medical Center, University of Ulsan College of Medicine, Seoul, ${ }^{2}$ Department of Anesthesiology and Pain Medicine, Seoul Teunteun Neurosurgery, Wonju, ${ }^{3}$ Department of Anesthesiology and Pain Medicine, CHA Bundang Medical Center, CHA University, Seongnam, Korea

Background: Generally, lactate levels $>2 \mathrm{mmol} / \mathrm{L}$ represent hyperlactatemia, whereas lactic acidosis is often defined as lactate $>4 \mathrm{mmol} / \mathrm{L}$. Although hyperlactatemia is common finding in liver transplant (LT) candidates, association between lactate and organ failures with Acute-on-chronic Liver Failure (ACLF) is poorly studied. We searched the important variables for pre-LT hyperlactatemia and examined the impact of preoperative hyperlactatemia on early mortality after LT.

Methods: A total of 2,002 patients from LT registry between January 2008 and February 2019 were analyzed. Six organ failures (liver, kidney, brain, coagulation, circulation, and lung) were defined by criteria of EASL-CLIF ACLF Consortium. Variable importance of preoperative hyperlactatemia was examined by machine learning using random survival forest (RSF). Kaplan-Meier Survival curve analysis was performed to assess 90-day mortality.

Results: Median lactate level was $1.9 \mathrm{mmol} / \mathrm{L}$ (interquartile range: 1.4, $2.4 \mathrm{mmol} / \mathrm{L}$ ) and 107 (5.3\%) patients showed $>4.0 \mathrm{mmol} / \mathrm{L}$. RSF analysis revealed that the four most important variables for hyperlactatemia were MELD score, circulatory failure, hemoglobin, and respiratory failure. The 30-day and 90-day mortality rates were $2.7 \%$ and $5.1 \%$, whereas patients with lactate $>4.0 \mathrm{mmol} / \mathrm{L}$ showed increased rate of $15.0 \%$ and $19.6 \%$, respectively.

Conclusions: About $50 \%$ and $5 \%$ of LT candidates showed pre-LT hyperlactatemia of $>2.0$ $\mathrm{mmol} / \mathrm{L}$ and $>4.0 \mathrm{mmol} / \mathrm{L}$, respectively. Pre-LT lactate $>4.0 \mathrm{mmol} / \mathrm{L}$ was associated with increased early post-LT mortality. Our results suggest that future study of correcting modifiable risk factors may play a role in preventing hyperlactatemia and lowering early mortality after LT.

Keywords: Early mortality; Lactate; Liver transplantation; Random survival.

\section{Corresponding author}

Department of Anesthesiology and

Pain Medicine, Laboratory for Cardiovascular Dynamics, Asan Medical Center, University of Ulsan College of Medicine, 88 Olympic-ro 43-gil, Songpa-gu, Seoul 05505, Korea

Tel: 82-2-3010-3989

Fax: 82-2-470-1363

E-mail: kshwang@amc.seoul.kr

\section{INTRODUCTION}

Serum lactate level has been a useful tool for assessing se- verity of various diseases and is associated with poor outcomes in critically ill patients, such as septic, cardiogenic, hypovolemic shock, and liver failure [1-3]. Patients with end-

This is an Open Access article distributed under the terms of the Creative Commons Attribution Non-Commercial License (http://creativecommons.org/licenses/by-nc/4.0) which permits unrestricted non-commercial use, distribution, and reproduction in any medium, provided the original work is properly cited.

Copyright (c) the Korean Society of Anesthesiologists, 2021 
stage liver disease are prone to develop acute decompensation with progressive organ failures, which may lead to developing the acute-on-chronic liver failure (ACLF) [4].

However, relationships between pre-liver transplant (LT) lactate and liver disease severity with organ failures are poorly studied in LT candidates.

In the current study, we sought to comprehend the distribution of lactate in LT candidates and searched the risk factors for hyperlactatemia. Specifically, to identify the most susceptible organ failures for the development of hyperlactatemia, we employed random survival forest (RSF) analysis and definitions from the Chronic Liver Failure Consortium ACLF score (CLIF-C ACLFs) [5]. Random forest is the machine learning technique using ensemble learning method for classification, regression, survival and identifying important risk factors [6-8]. Additionally, we evaluated association between pre-LT hyperlactatemia and early mortality after LT.

\section{MATERIALS AND METHODS}

\section{Study population}

The study design and a waiver of informed consent for participants were approved by the Institutional Review Board (no. 2021-0711). Data was extracted from the institution's LT Registry, which prospectively registered patients who underwent LT. From January 2008 to February 2019, there were 4,406 potentially eligible LT recipients. Patients with toxic hepatitis $(\mathrm{n}=141)$, fulminant hepatic failure $(\mathrm{n}=$ 134), were excluded. The patients who did not measure lactate within 7 days before LT $(n=1,822)$ and those with incomplete data $(n=307)$ were also excluded. After exclusion, 2,002 LT recipients were finally included. We included the majority of heterogenous LT recipients except acute toxic or fulminant hepatitis because we aimed to identify which comorbidities, or organ failures were most susceptible to preLT hyperlactatemia.

\section{Data collection and definition of organ failures}

Patient demographics, medical history Model for Endstage Liver Disease score (MELDs), and laboratory variables were obtained automatically using a fully computerized data extraction software. Mortality data were obtained from patients' electronic medical records and the updated record of the institution's LT registry. To identify risk factors associ- ated with hyperlactatemia, we thoroughly investigated the association of hyperlactatemia with comorbidities and six organ failures (liver, kidney, brain, coagulation, circulation, and lungs) incorporated in definitions from the CLIF-C ACLFs. Briefly, they were defined as follows; Liver failure: bilirubin level of > $12 \mathrm{mg} / \mathrm{dl}$, Kidney failure: creatinine > $2.0 \mathrm{mg} / \mathrm{dl}$ or renal replacement, Brain failure: hepatic encephalopathy grade by West-Haven, 3-4, Coagulation failure: INR $\geq 2.5$, Circulatory failure: use of vasopressor, $\mathrm{Re}$ spiratory failure: $\mathrm{PaO}_{2} / \mathrm{FiO}_{2} \leq 200 ; \mathrm{SpO}_{2} / \mathrm{FiO}_{2} \leq 214$; or on ventilator treatment [5].

\section{Classification of patients with Lactate $>\mathbf{4 . 0}$ $\mathrm{mmol} / \mathrm{L}$ and $\leq 4.0 \mathrm{mmol} / \mathrm{L}$}

Among patients with $>1$ pre-transplant lactate measurements, the most proximate to the date of transplant within seven pre-transplant days was used. Lactate levels greater than $2 \mathrm{mmol} / \mathrm{L}$ represent hyperlactatemia, whereas lactic acidosis is generally defined as a serum lactate concentration above $4 \mathrm{mmol} / \mathrm{L}$.

We chose threshold of lactate as $4.0 \mathrm{mmol} / \mathrm{L}$ according to the Surviving Sepsis Campaign guidelines, in which A lactate $>4 \mathrm{mmol} / \mathrm{L}$ qualifies for administration of early quantitative resuscitation therapy [9].

\section{Statistical analysis}

Data were expressed as mean with standard deviation (SD) or median with interquartile range (IQR) for continuous variables, and numbers and percentages for categorical variables. Analyses between groups were performed using Student's $t$-test, the Mann-Whiney $U$ test, analysis of variance, or the Kruskal-Wallis test for continuous variables and the $\chi^{2}$ test or Fisher's exact test for categorical variables, as appropriate. Kaplan-Meier survival curve analysis with logrank test was used to evaluate the risk of 90-day mortality.

\section{Identifying important variables for preoperative hyperlactatemia}

Variable importance (VIMP) was defined in classification and regression trees of RSF using a measure involving surrogate variables. Since VIMP is the difference between Out-ofBag prediction error before and after permutation, a large VIMP value indicates that misspecification detracts from the variable predictive accuracy in the forest. 
A random forest for pre-LT serum lactate levels was generated by creating 1,000 trees using ggRandomForest R-software package. The following variables were included for predicting preoperative hyperlactatemia: MELDs, left ventricular ejection fraction, hemoglobin, sepsis, and six organ failures defined by CLIF-C ACLFs.

All statistical analyses were performed using $\mathrm{R}$ version 3.6.1 (R Foundation for Statistical Computing, Austria). P value of less than 0.05 was considered statistically significant.

\section{RESULTS}

Of 2,002 LT recipients included, their mean age was 53.0 $(48.0,58.0)$ years and $1,489(74.4 \%)$ were men. The mean MELDs was $16.0(10.0,26.0)$. The primary causes of liver disease were $\mathrm{B}$ or $\mathrm{C}$ virus-related liver cirrhosis (69.2\%), alcoholic liver disease (19.8\%) and others (11.0\%) (Table 1). Prevalence of organ failures defined by CLIF-C ACLFs [5] were liver failure $(24.0 \%)$, respiratory failure (10.8\%), kidney failure $(8.3 \%)$, coagulation failure $(9.3 \%)$, circulatory failure (5.6\%), and brain failure (2.6\%), respectively (Table 2).

\section{Distribution of Lactate in LT candidates}

Median lactate level was $1.9 \mathrm{mmol} / \mathrm{L}$ (range: 0.4-24.6, IQR: 1.4, $2.4 \mathrm{mmol} / \mathrm{L})$ and 107 (5.3\%) patients showed lactate $>4.0 \mathrm{mmol} / \mathrm{L}$. When patients were grouped with MELDs of < 15, 15-35, > 35, their median lactate level were $1.7(1.4,2.2), 1.9(1.5,2.5)$, and $2.4(1.9,3.6) \mathrm{mmol} / \mathrm{L}$, respectively $(\mathrm{P}<0.001)$ (Fig. 1).

\section{Important variables for pre-LT hyperlactatemia}

When we ignored the negative value of VIMP, the four most important variables for hyperlactatemia were MELDs, circulatory failure, hemoglobin, and respiratory failure, respectively (Fig. 2).

Table 1. Descriptive Statistics by Lactate $4.0 \mathrm{mmol} / \mathrm{L}$

\begin{tabular}{|c|c|c|c|c|}
\hline Variable & Lactate $\leq 4 \mathrm{mmol} / \mathrm{L}(\mathrm{n}=1,895)$ & Lactate $>4 \mathrm{mmol} / \mathrm{L}(\mathrm{n}=107)$ & Total $(n=2,002)$ & $P$ value \\
\hline Sex, male & $1,408(74.3)$ & $81(75.7)$ & $1,489(74.4)$ & 0.834 \\
\hline Age (yr) & $53.0(48.0,58.0)$ & $53.0(46.5,57.0)$ & $53.0(48.0,58.0)$ & 0.563 \\
\hline $\mathrm{BMI}\left(\mathrm{kg} / \mathrm{m}^{2}\right)$ & $24.0(21.9,26.5)$ & $23.7(21.0,26.3)$ & $24.0(21.8,26.4)$ & 0.058 \\
\hline Re-transplantation & $77(4.1)$ & $14(13.1)$ & $91(4.5)$ & $<0.001^{\dagger}$ \\
\hline OLT & $326(17.2)$ & $58(54.2)$ & $384(19.2)$ & $<0.001^{\dagger}$ \\
\hline CAD & $79(6.3)$ & $8(12.1)$ & $87(6.6)$ & 0.112 \\
\hline CVA & $15(0.9)$ & $1(1.2)$ & $16(0.9)$ & 1.000 \\
\hline Diabetes mellitus & $459(24.2)$ & $29(27.1)$ & $488(24.4)$ & 0.576 \\
\hline Hypertension & $273(14.4)$ & $17(15.9)$ & $290(14.5)$ & 0.778 \\
\hline MELD & $15.0(10.0,24.0)$ & $34.0(21.0,41.0)$ & $16.0(10.0,26.0)$ & $<0.001^{\dagger}$ \\
\hline \multicolumn{5}{|l|}{ Causes of liver disease } \\
\hline Hepatitis B virus & $1192(62.9)$ & $54(50.5)$ & $1246(62.2)$ & $0.013 *$ \\
\hline Hepatitis C virus & $135(7.1)$ & $6(5.6)$ & $141(7.0)$ & 0.687 \\
\hline Alcohol & $366(19.3)$ & $31(29.0)$ & $397(19.8)$ & $0.021 *$ \\
\hline Others & $202(10.7)$ & $16(14.9)$ & $218(11.0)$ & 0.853 \\
\hline \multicolumn{5}{|l|}{ Laboratory values } \\
\hline Hemoglobin (g/dl) & $10.3(8.8,12.2)$ & $9.0(8.0,10.2)$ & $10.3(8.7,12.2)$ & $<0.001^{\dagger}$ \\
\hline WBC $\left(x 10^{3} / \mu \mathrm{l}\right)$ & $3.6(2.4,5.2)$ & $7.1(4.2,11.8)$ & $3.7(2.5,5.5)$ & $<0.001^{\dagger}$ \\
\hline Natrium (mmol/L) & $138.0(135.0,141.0)$ & $137.0(134.0,141.5)$ & $138.0(135.0,141.0)$ & 0.954 \\
\hline $\mathrm{CRP}(\mathrm{mg} / \mathrm{dl})$ & $0.4(0.1,1.3)$ & $1.7(0.7,3.6)$ & $0.4(0.1,1.4)$ & $<0.001^{\dagger}$ \\
\hline INR & $1.4(1.2,1.8)$ & $2.1(1.5,2.6)$ & $1.5(1.2,1.9)$ & $<0.001^{\dagger}$ \\
\hline Total bilirubin (mg/dl) & $2.4(1.2,9.2)$ & $15.4(4.8,30.2)$ & $2.6(1.3,11.1)$ & $<0.001^{\dagger}$ \\
\hline Creatinine (mg/dl) & $0.8(0.6,1.0)$ & $1.2(0.7,2.1)$ & $0.8(0.7,1.0)$ & $<0.001^{\dagger}$ \\
\hline Lactate (mmol/L) & $1.8(1.4,2.3)$ & $5.5(4.6,7.0)$ & $1.9(1.4,2.4)$ & $<0.001^{\dagger}$ \\
\hline
\end{tabular}

Values are presented as median (1Q, 3Q) or number (\%). BMI: body mass index, OLT: orthotopic liver transplantation, CAD: coronary artery disease, CVA: cerebrovascular accident, MELD: model for end-stage liver disease, WBC: white blood cell, CRP: C-reactive protein, INR: international normalized ratio. ${ }^{*} \mathrm{P}$ value $<0.05,{ }^{\dagger} \mathrm{P}$ value $<0.01$. 
Table 2. CLIF-C ACLFs Organ Failures by Lactate $4.0 \mathrm{mmol} / \mathrm{L}$

\begin{tabular}{lcccc}
\hline \multicolumn{1}{c}{ Variable } & Lactate $\leq 4 \mathrm{mmol} / \mathrm{L}(\mathrm{n}=1,895)$ & Lactate $>4 \mathrm{mmol} / \mathrm{L}(\mathrm{n}=107)$ & Total $(\mathrm{n}=2,002)$ & $\mathrm{P}$ value \\
\hline CLIF_liver & $413(21.8)$ & $67(62.6)$ & $480(24.0)$ & $<0.001^{\dagger}$ \\
CLIF_respiratory & $162(8.6)$ & $55(51.4)$ & $217(10.8)$ & $<0.001^{\dagger}$ \\
CLIF_kidney & $138(7.3)$ & $28(26.2)$ & $166(8.3)$ & $<0.001^{\dagger}$ \\
CLIF_coagulation & $157(8.3)$ & $29(27.1)$ & $186(9.3)$ & $<0.001^{\dagger}$ \\
CLIF_circulatory & $77(4.1)$ & $36(33.6)$ & $113(5.6)$ & $52(2.6)$ \\
CLIF_brain & $41(2.2)$ & $11(10.3)$ & $0.001^{\dagger}$ \\
\hline
\end{tabular}

Values are presented as number (\%). CLIF-C ACLFs: chronic liver failure consortium acute-on-chronic liver failure score, CLIF: chronic liver failure. ${ }^{\dagger} \mathrm{P}$ value $<0.01$

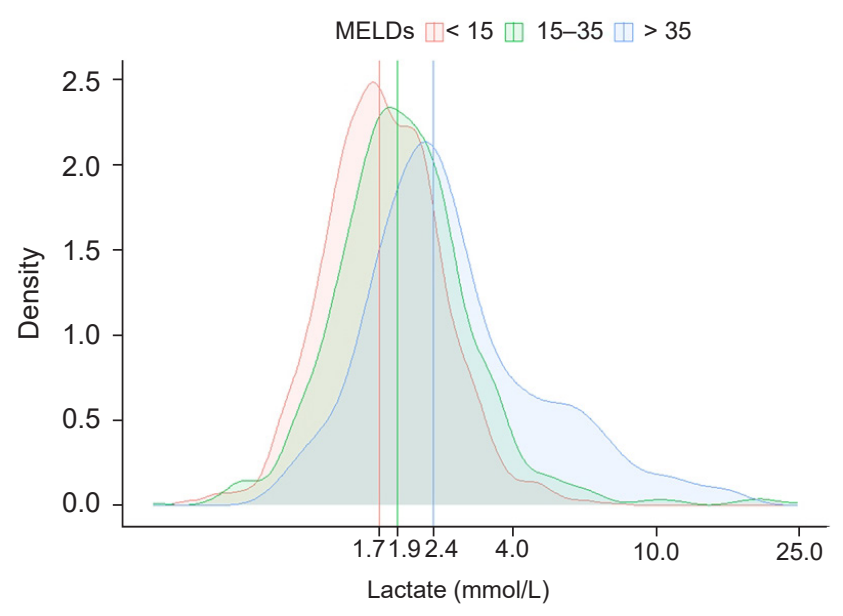

Fig. 1. Density histogram of pre-liver transplant lactate is showing rightward shift, according to MELDs classification of < 15, 15-35, $>$ 35. MELDs: model for end-liver disease score.

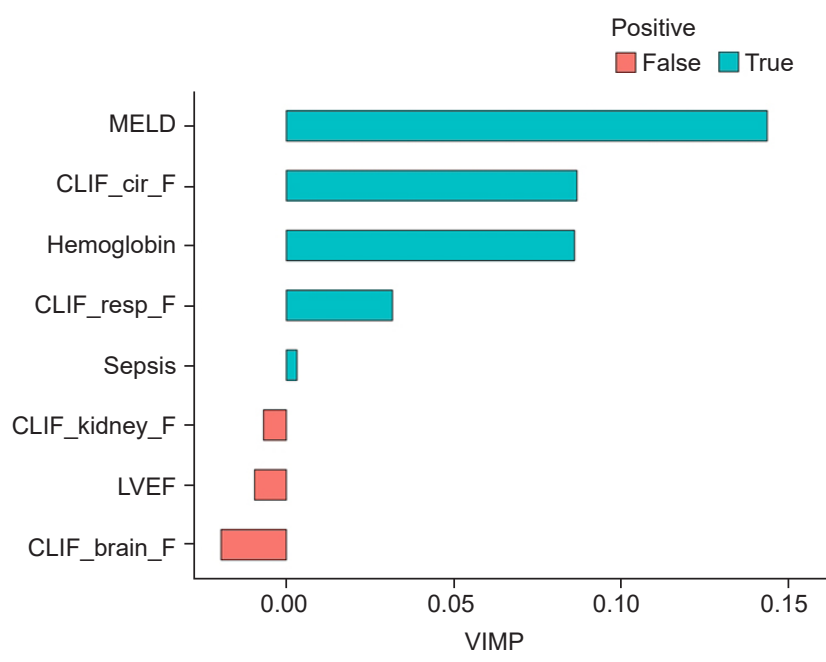

Fig. 2. Random forest variable importance (VIMP). Blue bars indicate positive VIMP, red indicates negative VIMP. Importance is relative to positive length of bars. VIMP: variable importance, MELD: model for end-liver disease, CLIF_cir_F: circulatory failure by CLIF score, CLIF_resp_F: respiratory failure by CLIF score, CLIF_kidney_F: kidney failure by CLIF score, LVEF: left ventricular ejection fraction, CLIF_brain_F: brain failure by CLIF score.

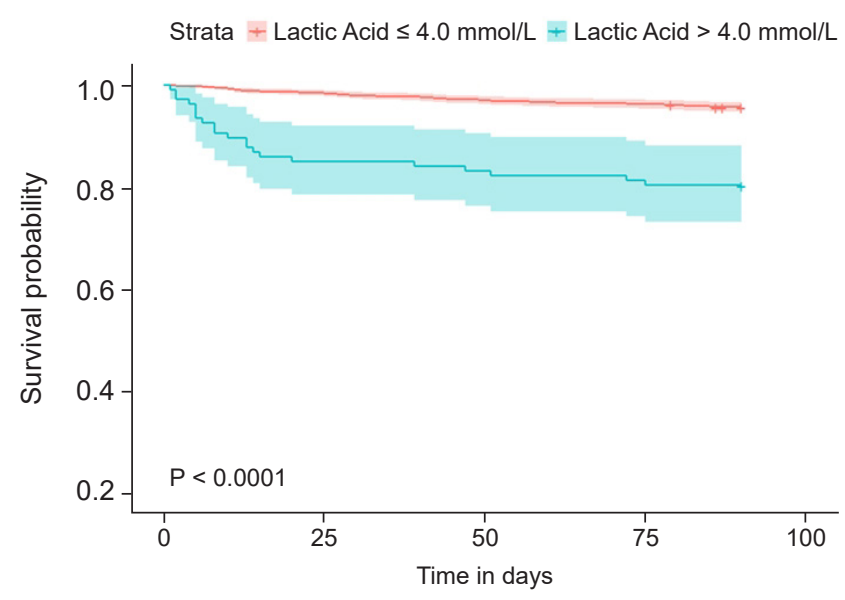

Fig. 3. The 90-day Kaplan-Meier survival curve stratified by lactate $4.0 \mathrm{mmol} / \mathrm{L}$ with shaded $95 \%$ confidence bands.

\section{Survival analysis on 30-day and 90-day mortality}

The 30-day and 90-day mortality rates were $2.7 \%$ and $5.1 \%$, however, patients with lactate $>4.0 \mathrm{mmol} / \mathrm{L}$ showed increased morality rate of $15.0 \%$ and $19.6 \%$, respectively. Kaplan-Meier analysis showed clear separation of survival curve of 90-day mortality $(\mathrm{P}<0.001$, Fig. 3).

\section{DISCUSSION}

Lactate levels is known to be a useful and rapid tool for assessing severity of disease in critically ill patients [10]. It has been shown that increased lactate levels and reduced lactate clearance are associated with mortality in critically ill patients with liver cirrhosis [1]. Therefore, lactate is believed to be a simple and accurate prognostic marker, and its incorporation improved performance of CLIF-C ACLFs significantly [10]. The Asian Pacific Association for the Study of the Liver (APASL) also incorporated lactate levels in its APASL 
ACLF research consortium-ACLF score [11].

Conventionally, lactate has been considered as a marker for tissue hypoxia, as lactate is overproduced and underutilized under anaerobic glycolysis due to impaired mitochondrial oxidation [12]. Therefore, increased serum lactate is usually the result from an imbalance of increased lactate production or reduced consumption [12]. Given that the liver is responsible for about $70 \%$ of whole-body lactate clearance, especially acute and fulminant hepatic impairment is associated with increased lactate levels and poor outcomes [13]. In contrast, under stable conditions, even severe liver cirrhosis has rarely been known to be associated with relevantly elevated lactate levels [12].

In the current study, after exclusion of toxic and fulminant hepatic failure, we investigated the important variables for preoperative hyperlactatemia in LT candidates and the relation between preoperative hyperlactatemia and early outcome after LT by using random forest survival analysis. We found that lactate monitoring is important in LT candidates because patients with lactate $>4.0 \mathrm{mmol} / \mathrm{L}$ showed increased 30-day morality rate of $15.0 \%$ and 90 -day mortality rate of $19.6 \%$, respectively.

Although the lower limit of the normal range for the blood lactate level, $0.5 \mathrm{mmol} / \mathrm{L}$, is generally accepted among clinical laboratories, the upper limit can vary considerably, from $1.0 \mathrm{mmol} / \mathrm{L}$ to $2.2 \mathrm{mmol} / \mathrm{L}$ [12]. However, a plasma lactate concentration that exceeds $4 \mathrm{mmol} / \mathrm{L}$ generally defines lactic acidosis, even among patients without systemic acidemia. In the current study $5.3 \%$ of LT candidates fell into this category of lactate $>4 \mathrm{mmol} / \mathrm{L}$.

The important variables related to hyperlactatemia were MELDs, circulatory failure, hemoglobin level, and respiratory failure in the order of importance. MELD score is useful for estimating overall disease severity and predicting survival in the patient with liver disease [14]. Therefore, the highest VIMP of MELD score in the current study may be related to hepatic dysfunction and reduced metabolism of lactate. Another important thing to be considered in the VIMP analysis is that circulatory failure and hemoglobin were chosen as VIMP in the current study. These results suggest that not only liver disease severity but also circulatory disturbance with anemia is an important contributor of pre-LT hyperlactatemia.

Circulatory failure has been defined typically as a condition in which the circulation is insufficient to deliver adequate oxygen to match the needs of the oxidizing tissues. However, the correlation between cardiac index and hyper- lactatemia is not noticeably clear. A study reported that lactate levels were normal in about $75 \%$ of the patients with advanced heart failure [15], however, in LT candidates, circulatory failure defined by CLIF-C ACLFs remained one of important risk factors for pre-LT hyperlactatemia in the current study.

Both anemia and hypotension may induce tissue hypoxia due to reduced tissue oxygen delivery. In our study, patients who received transfusions or vasopressors would have higher plasma lactate concentrations due to anemia or hypotension. However, the retrospective nature of the current study cannot discriminate that high lactate was associated with either transfusions or vasopressors, or both.

Variables of importance for pre-LT hyperlactatemia was identified by machine learning using RSF in the current study. One of advantages of this analysis is that missing data are also less of a concern when using RSF. RSF performs excellently even with heavy missingness and when missing data are not missing completely at random [16,17]. Of 2,002 cases of lactic acid analysis, missing values were organ failures of 4 , sepsis of 1 , and left ventricular ejection fraction of 53 cases in the current study, respectively.

In this analysis, VIMP close to zero indicates the variable contributes nothing to predictive accuracy, and negative values indicate the predictive accuracy improves when the variable is misspecified. Therefore, we ignored variables with negative and near zero values of VIMP.

This study has several limitations. The enrolled patients are from the observational cohort study in a single center and retrospective observational design. Therefore, further prospective randomized control studies are warranted to validate our results.

In conclusion, about $50 \%$ and $5 \%$ of LT candidates showed pre-LT hyperlactatemia of $>2.0 \mathrm{mmol} / \mathrm{L}$ and $>4.0 \mathrm{mmol} / \mathrm{L}$, respectively and those with lactate $>4.0 \mathrm{mmol} / \mathrm{L}$ was associated with increased early post-LT mortality. Four the most important variables for hyperlactatemia were MELD score, circulatory failure, hemoglobin, and respiratory failure. Our results suggest that future study of correcting modifiable risk factors, such as anemia and hypotension correction, may play a role in preventing hyperlactatemia and lowering early mortality after LT.

\section{ACKNOWLEDGEMENTS}

This research was supported by a grant of the Korea Health Technology R\&D Project through the Korea Health 
Industry Development Institute (KHIDI), funded by the Ministry of Health \& Welfare, Republic of Korea (grant number: HI18C2383).

\section{CONFLICTS OF INTEREST}

No potential conflict of interest relevant to this article was reported.

\section{DATA AVAILABILITY STATEMENT}

The datasets generated during and/or analyzed during the current study are available from the corresponding author on reasonable request.

\section{AUTHOR CONTRIBUTIONS}

Conceptualization: Yuseon Cheong, Gyu-Sam Hwang. Data curation: Yuseon Cheong, Kyoung-Sun Kim. Formal analysis: Yuseon Cheong, Bo-Hyun Sang. Funding acquisition: Gyu-Sam Hwang. Methodology: Bo-Hyun Sang. Project administration: Gyu-Sam Hwang. Visualization: Sangho Lee. Writing - original draft: Yuseon Cheong, Sangho Lee, Do-Kyeong Lee. Writing -review \& editing: Gyu-Sam Hwang. Investigation: Kyoung-Sun Kim Resources: Sangho Lee. Software: Do-Kyeong Lee. Supervision: Gyu-Sam Hwang. Validation: Kyoung-Sun Kim.

\section{ORCID}

Yuseon Cheong, http://orcid.org/0000-0001-7662-7158

Sangho Lee, http://orcid.org/0000-0001-5943-7715

Do-Kyeong Lee, http://orcid.org/0000-0001-6176-597X

Kyoung-Sun Kim, http://orcid.org/0000-0002-6643-9177

Bo-Hyun Sang, http://orcid.org/0000-0001-6890-4629

Gyu-Sam Hwang, http://orcid.org/0000-0002-3627-1107

\section{REFERENCES}

1. Cardoso FS, Abraldes JG, Sy E, Ronco JJ, Bagulho L, Mcphail MJ, et al. Lactate and number of organ failures predict intensive care unit mortality in patients with acute-on-chronic liver failure. Liver Int 2019; 39: 1271-80.

2. Sundaram V, Jalan R, Wu T, Volk ML, Asrani SK, Klein AS, et al. Factors associated with survival of patients with severe acuteon-chronic liver failure before and after liver transplantation. Gastroenterology 2019; 156: 1381-91.e3.
3. Weiss SL, Peters MJ, Alhazzani W, Agus MSD, Flori HR, Inwald DP, et al. Surviving sepsis campaign international guidelines for the management of septic shock and sepsis-associated organ dysfunction in children. Intensive Care Med 2020; 46(Suppl 1): 10-67.

4. Jeppesen JB, Mortensen C, Bendtsen F, Møller S. Lactate metabolism in chronic liver disease. Scand J Clin Lab Invest 2013; 73: 293-9.

5. Jalan R, Saliba F, Pavesi M, Amoros A, Moreau R, Ginès P, et al. CANONIC study investigators of the EASL-CLIF Consortium. Development and validation of a prognostic score to predict mortality in patients with acute-on-chronic liver failure. J Hepatol 2014; 61: 1038-47.

6. Hsich E, Gorodeski EZ, Blackstone EH, Ishwaran H, Lauer MS. Identifying important risk factors for survival in patient with systolic heart failure using random survival forests. Circ Cardiovasc Qual Outcomes 2011; 4: 39-45.

7. Ishwaran H, Kogalur UB. Consistency of random survival forests. Stat Probab Lett 2010; 80: 1056-64.

8. O’Brien RC, Ishwaran H, Szczotka-Flynn LB, Lass JH; Cornea Preservation Time Study (CPTS) Group. Random survival forests analysis of intraoperative complications as predictors of descemet stripping automated endothelial keratoplasty graft failure in the cornea preservation time study. JAMA Ophthalmol 2021; 139: 191-7.

9. Levy MM, Evans LE, Rhodes A. The surviving sepsis campaign bundle: 2018 update. Intensive Care Med 2018; 44: 925-8.

10. Drolz A, Horvatits T, Rutter K, Landahl F, Roedl K, Meersseman $\mathrm{P}$, et al. Lactate improves prediction of short-term mortality in critically ill patients with cirrhosis: a multinational study. Hepatology 2019; 69: 258-69.

11. Choudhury A, Jindal A, Maiwall R, Sharma MK, Sharma BC, Pamecha V, et al. APASL ACLF Working Party. Liver failure determines the outcome in patients of acute-on-chronic liver failure (ACLF): comparison of APASL ACLF research consortium (AARC) and CLIF-SOFA models. Hepatol Int 2017; 11 $461-71$.

12. Kraut JA, Madias NE. Lactic acidosis. N Engl J Med 2014; 371: 2309-19

13. Macquillan GC, Seyam MS, Nightingale P, Neuberger JM, Murphy N. Blood lactate but not serum phosphate levels can predict patient outcome in fulminant hepatic failure. Liver Transpl 2005; 11: 1073-9.

14. Freeman RB Jr, Wiesner RH, Harper A, McDiarmid SV, Lake J, Edwards E, et al. UNOS/OPTN Liver Disease Severity Score, UNOS/OPTN Liver and Intestine, and UNOS/OPTN Pediatric Transplantation Committees. The new liver allocation system: 
moving toward evidence-based transplantation policy. Liver Transpl 2002; 8: 851-8.

15. Adamo L, Nassif ME, Novak E, LaRue SJ, Mann DL. Prevalence of lactic acidaemia in patients with advanced heart failure and depressed cardiac output. Eur J Heart Fail 2017; 19: 1027-33.
16. Ishwaran H, Gerds TA, Kogalur UB, Moore RD, Gange SJ, Lau BM. Random survival forests for competing risks. Biostatistics 2014; 15: 757-73.

17. Tang F, Ishwaran H. Random forest missing data algorithms. Stat Anal Data Min 2017; 10: 363-77. 